MATEC Web of Conferences 4, 02002 (2013)

DOI: $10.1051 /$ matecconf/20130402002

(C) Owned by the authors, published by EDP Sciences, 2013

\title{
Nanolayers for early diagnostics of proteins involved in degenerative amyloidosis
}

\author{
M.R. Martina, E. Mercatelli, P. Baglioni, and G. Caminati * \\ Department of Chemistry “Ugo Schiff”/CSGI, University of Florence, Via della Lastruccia 3-5, I-50019, Sesto Fiorentino, \\ Italy \\ E-mail: gabriella.caminati@unifi.it
}

\begin{abstract}
FK-506 binding protein (FKBP12) is a protein of the family of immunophilins, involved in many neurodegenerative diseases such as Alzheimer's syndrome where FKBP12 is known to be over-expressed in early stages of the disease. We designed and built Langmuir-Blodgett nanostructures incorporating ligands with high affinity for FKBP12: Tacrolimus (FK506) and Rifaximin as candidate nanosensors to detect low FKBP12 concentration in the initial phase of the amyloidosis. The binding process of the different ligands has been studied by means of photophysical measurements investigating the fluorescence quenching of the tryptophan residue in the binding pocket of FKBP12 by addition of the ligand in solution. Immobilization of the ligands was achieved adopting biomimetic strategy: phospholipid Langmuir-Blodgett films are proposed as nanoscaffolds for ligand inclusion. Several phospholipid nanoarchitectures differing in lipid composition, fluidity, number of layers and method of production (incubation versus co-spreading) were screened. The results have shown that both FK506 and Rifaximin ligands penetrate the lipid matrix either as monomers or as aggregates depending on their initial concentration. More importantly, the experiments demonstrated that the ligands in the LB scaffolds efficiently quench FKBP12 fluorescence in solution as a consequence of ligand binding to the protein.
\end{abstract}

\section{Introduction}

The FK506 binding proteins (FKBP) belong to a family of immunophilins with domains of protein-protein interactions and various cellular functions. A direct involvement of FKBP12 in neurodegenerative diseases such as Alzheimer and Parkinson diseases, Multiple Sclerosis proliferation disorders and cancer was recently proposed [1]. Furthermore, such studies evidenced two important aspects: a decrease of $\beta$-amyloid formation in neurodegenerative diseases when the FKBP12 protein is inhibited by efficient ligands and over expression of FKBP12 in the very early stages of such pathologies [1].

All FKBPs contain a peptidyl-prolyl isomerase domain (PPI) catalyzing cis-trans isomerization of proline aminoacids. The binding of immunosuppressive ligands, such as FK506, rapamycin and cyclosporine A, to this domain inhibits the PPIase activity and leads to immunosuppression by inhibition of the phosphatase activity of calcineurin, a protein involved in the activation pathway of T-cells. In the present work, we studied different approaches for the inclusion of ligands with high affinity for FKBP12 in biomimetic nanostructures, focusing in particular on planar phospholipid layers. The long-term goals of these studies include the design of nano-functionalized sensors for the detection of the FKBP12 protein in the initial phase of the amyloidosis as well as the study of optimal biomimetic systems for ligand delivery in the therapeutic stage. Eventually, investigation on the interaction mechanism of this class of ligands with FKPB12 in the presence of candidates for fibrillization, i.e. $\alpha$-synuclein, will provide an effective mean for elucidating the role of FKBP12 itself in amyloidogenesis [2,3]. Results in these directions will be presented in forthcoming studies.

In the present work, we examined two ligands of FKBP12, namely FK506, a natural ligand also known as Tacrolimus, and Rifaximin, an antibiotic molecule selected for its analogy of cyclic rigid structure with FK506. FK506, a 23-membered macrolide lactone (Fig.1a), is an immunosuppressive drug used in treating patients after organ transplant, whereas Rifaximin (Fig.1b) is a member of the rifamycins antibiotic family with broad-spectrum activity against Gram-positive bacteria. The action mechanism of these drugs is based on the inhibition of RNA synthesis and MRP (Multidrug Resistance-associated Protein) activity responsible for the failure of anti-cancer therapies [4]. 


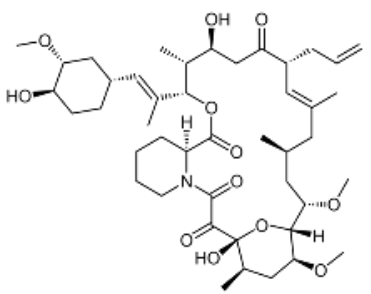

(a)

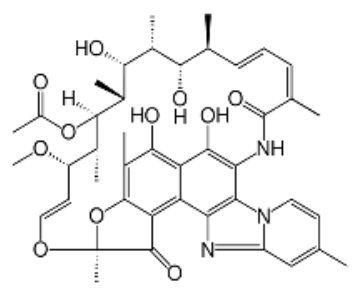

(b)

Fig. 1. Chemical structure of Tacrolimus (FK506) (a) and Rifaximin (b)

In particular, we report here a summary of the studies on Langmuir-Blodgett (LB) mono and bilayers, where incorporation of the ligand was accomplished either by immersion of the lipid layers in the ligand solution or by co-spreading in the preparation of the precursor monolayer. The inclusion of the ligand in the LB structure was ascertained by means of absorption and emission spectroscopy that allowed for the description of the dependence of ligand uptake on bulk concentration, LB sequence, layer number and phospholipid composition of the layers.

Fluorescence quenching of FKBP12 in the presence of LB scaffold with immobilized ligands was also studied, the results enabled to assess the occurrence of a binding process at the outer layer of the LB nanostructures.

\section{Materials and methods}

Dipalmitoyl phosphatidylglycerol sodium salt (DPPG-Na, purity >99\%) and Tacrolimus (FK506) were supplied by Sigma, Dipalmitoyl-sn-glycero-3phosphocholine (DPPC) and Palmitoyl Oleoyl-snglycero-3-phosphoglycerol sodium salt (POPG) were supplied by Avanti Polar Lipids, Rifaximin (Rfx, purity 99\%) was a gift from FATRO-Pharmaceutical Veterinary Industry (Bologna, Italy). Chloroform/methanol (10:1 $\mathrm{v} / \mathrm{v})$ mixtures were used for DPPG-Na spreading solutions; Sigma Aldrich (Italy) supplied chloroform, methanol and dimethylsulfoxide (DMSO). Stock solutions of FK506 and Rifaximin were prepared dissolving the ligands in dimethylsulfoxide. Water was obtained from a Milli-RO coupled with a Milli-Q set-up

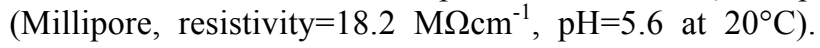
Phosphate buffer 0.1 M (pH=7.4, 0.15 M NaCl), used for aqueous solutions was prepared using di-potassium hydrogen phosphate $\left(\mathrm{K}_{2} \mathrm{HPO}_{4}\right)$ and sodium dihydrogen phosphate $\left(\mathrm{NaH}_{2} \mathrm{PO}_{4}\right)$, purchased from Fluka. QS quartz slides (Hellma, Germany) were used as solid supports for LB films.

LB films were prepared using a KSV 3000 film balance apparatus (KSV, Finland). DPPG-Na and DPPC/POPG (8:2) + FK506 0.29\% mixture were spread from chloroform/methanol solution on phosphate buffer subphase $\left(\mathrm{T}=25^{\circ} \mathrm{C}, \mathrm{pH}=7.4\right)$. Twenty minutes were allowed for solvent evaporation before monolayer compression. The compression rate was $6 \mathrm{~mm} / \mathrm{min}^{-1}$. Monolayers were transferred from water-air interface to the solid slides at constant surface pressure, 20 min were allowed for monolayer equilibration at the selected target surface pressure. The dipper rate was $3 \mathrm{~mm} / \mathrm{min}$ for LB transfer. Incorporation of Rfx in the transferred DPPG-Na layers was achieved incubating the LB films in aqueous solution (water/DMSO mixtures 50:1 v/v) of the antibiotic. Incorporation of FK506 was also achieved by co-spreading of DPPC/POPG (8:2) + FK506 0.29\% mixture in chloroform/methanol $(10: 1 \mathrm{v} / \mathrm{v})$. Absorption spectra were recorded on a Lambda900 spectrophotometer (Perkin Elmer, Italy) with cuvette cell holder connected to a Haacke thermostatic bath. Fluorescence spectra were recorded on a LS50B spectrofluorimeter (Perkin Elmer, Italy), excitation and emission slits were set to 3.5 or $5 \mathrm{~nm}$ depending on the optical path (OP) length of the cell employed for solutions and $4 \mathrm{~nm}$ for LB films. QS cells with $0.3 \mathrm{~cm}$ optical path length from Hellma (Hellma GmbH \& Co. $\mathrm{KG}$, Mulheim, Germany) were used for quenching experiments, whereas $1 \mathrm{~cm}$ OP cuvettes were used for absorption measurements of solutions and for absorption and fluorescence measurements of LB films. Cuvettes and quartz slides were cleaned with piranha solution and carefully rinsed with water and ethanol. The cuvettes were dried by nitrogen flushing prior to each measurement. All fluorescence measurements were run at $15^{\circ} \mathrm{C}$ unless otherwise stated in the text, a temperature probe was inserted in the cuvette to ensure optimal temperature control. The concentration of stock FKBP12 solutions in PBS buffer $(\mathrm{pH}=7.4,0.15 \mathrm{M} \mathrm{NaCl})$ was determined by UV/Vis absorption $\left(\varepsilon_{280}=9970 \mathrm{M}^{-1} \mathrm{~cm}^{-1}\right)$. Aliquots of stock solution of ligands were used to prepare adequate concentrations for quenching experiments by dilution with standard phosphate buffer. Fluorescence quenching assays were performed measuring the decrease of intrinsic tryptophan fluorescence at $330 \mathrm{~nm}$ as function of ligand concentration $\left(\lambda_{\mathrm{exc}}=280 \mathrm{~nm}\right)$. The final FKBP12 concentration used for all measurements was $1 \mu \mathrm{M}$. The desired ligand concentration in the sample was obtained by appropriate dilution of ligand stock solution. The emission spectrum of the corresponding blank solution containing only the solvent was always recorded separately and subtracted. In all cases the final DMSO concentration in the sample was kept lower than $0.3 \%$ v/v. Emission spectra of the corresponding FKBP12-free samples were subtracted from the corresponding FKBP12-ligand fluorescence spectra. The apparent dissociation constant for each ligand was calculated from the decrease of fluorescence intensity values at $\lambda_{\mathrm{em}}=325$ $\mathrm{nm}$ as function of ligand concentration as previously reported [5].

\section{Results}

The binding process of the ligands was preliminary studied by means of photophysical measurements in solution investigating the fluorescence quenching of the tryptophan residue in the binding pocket of FKBP12 [6]. An efficient fluorescence quenching of FKBP12 was observed upon addition of the ligands in solution. The dissociation constants, $\mathrm{K}_{\mathrm{d}}$, for FK506 and Rfx were calculated according to literature and recent studies in our group $[7,8]$; the results are reported in table 1 together 
with the $\mathrm{K}_{\mathrm{d}}$ value obtained for Rapamycin, a well known high affinity compound for FKBP12, using literature experimental data [8] for the fitting. Interestingly, the data reveal that Rfx binds almost as efficiently as FK506 to the protein.

Tab.1. Dissociation constants, $K_{d}$ of examined ligands

\begin{tabular}{|c|c|}
\hline Ligands & $\mathbf{K}_{\mathbf{d}}(\mathbf{n M})$ \\
\hline FK506 & $6.2 \pm 0.2$ \\
\hline Rifaximin & $13.8 \pm 0.3$ \\
\hline Rapamycin & $5.5 \pm 0.2$ \\
\hline
\end{tabular}

The ligands were embedded in a planar scaffold using a biomimetic approach: that is to say preparing Langmuir-Blodgett films of phospholipids. The optimal phospholipid composition for each ligand has been established from the analysis of previous literature [9] combined with our own experimental work.

We examined two different inclusion procedures: i) incubation of LB films in solutions of the ligand at different concentrations or ii) inclusion of the ligand by co-spreading with the phospholipid. Best inclusion results for Rfx were obtained using the inclusion procedure with LB layers of pure DPPG transferred at $\pi_{\mathrm{tr}}=25 \mathrm{mNm}^{-1}$ as shown also in other works [10]. Tuning of draining time and slide speed, resulted in transfer ratios (TR) close to unity for the upstroke and $\mathrm{TR}=0$ for the downstroke suggesting the formation of 2 z-type LB layers [11].

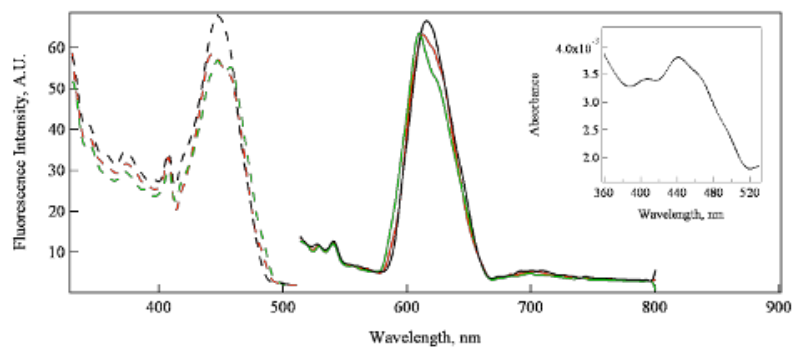

Fig. 2. Fluorescence excitation (dotted line) and emission (solid line) spectra of DPPG LB films incubated in $[\mathrm{Rfx}]=1 \times 10^{-5} \mathrm{M}$ (red), $[\mathrm{Rfx}]=0.8 \times 10^{-5} \mathrm{M}$ (green), $[\mathrm{Rfx}]=1 \times 10^{-6} \mathrm{M}$ (black).

$\lambda_{\text {exc }}=450 \mathrm{~nm}$ and $\lambda_{\text {em }}=620 \mathrm{~nm}$. Inset: UV-Vis spectra of DPPG LB film incubated in Rfx.

Fluorescence emission and excitation spectra (see figure 2) for one DPPG LB layer incubated in Rfx solution unambiguously evidence the inclusion of the ligand in the nanolayer. This assignment is further supported by the presence of the characteristic absorption band at $450 \mathrm{~nm}$ of Rfx in the UV-Vis spectra of the same system (inset fig. 2). Increasing $\mathrm{Rfx}$ concentration results in a corresponding increase of absorption paralleled by a slight decrease in fluorescence emission, a behaviour that can be correlated to self-quenching phenomena. Conversely, for z-type DPPG film, incubation with Rfx induces only a minor increase in the absorption and emission signal compared to 1 LB layer, suggesting a preferential localization of the ligand in the outer layer of the LB films, extensive characterization of these systems.
In the case of FK506 both procedures were tested: incubation against co-spreading. UV-Vis absorption spectra of incubated LB films showed that saturation of FK506 in the LB film was reached at high bulk concentrations of the ligand $\left(6 \times 10^{-5} \mathrm{M}\right)$, suggesting poor transfer from the bulk phase. Along the same lines, absorbance measurements of the depleted ligand solution after incubation confirmed that the overall uptake was much higher for Rfx than for FK506 with this procedure. Therefore, FK506 was co-spread at the water-air interface with a DPPC/POPG (8:2) mixture [9]. Spreading isotherms for monolayers at water-air interface obtained for co-spreading with $0.29 \%$ FK506 are reported in fig. 3 together with the results for pure DPPC/POPG 8:2 mixture. The difference in area between the two isotherms was calculated for several surface pressures providing an average value of $31 \AA^{2} /$ molecule. This difference refers to the contribution of FK506 in the monolayer, since this value is uniform in the entire pressure range screened, we conclude that FK506 penetrates the lipid matrix and safely exclude migration of the ligand from the monolayer to the subphase also in the condensed phase chosen for LB film transfer.

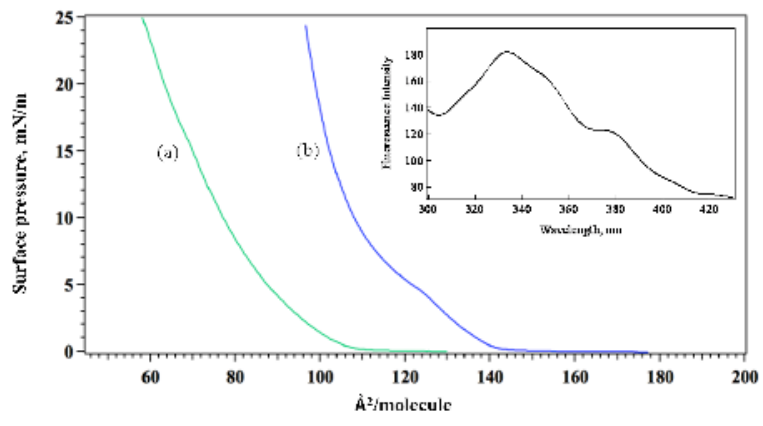

Fig. 3. Spreading isotherms of DPPC/POPG 8:2 (a) and DPPC/POPG 8:2 with FK506 (0.29\%) (b). Inset: fluorescence emission spectrum of FK506 in 1 LB layer of DPPC/POPG 8:2.

Monolayers obtained from co-spreading were successfully transferred onto quartz slides and further characterized collecting their fluorescence emission. The spectra reveal the specific FK506 emission band at 330 $\mathrm{nm}$ (see inset Fig. 3) confirming the presence of the ligand in the LB scaffolds.

FKBP12-ligands interactions in the LB nanolayers were assessed from fluorescence quenching experiments in analogy to bulk solution studies. Fluorescence spectra were recorded for the FKBP12 protein solution both alone and in the presence of LB nanolayers containing the ligands. Typical results, reported in Figure 4 for 1 LB layer, show an efficient fluorescence quenching of FKBP12 fluorescence with both ligands. Higher quenching was found for the Rfx ligand (Fig. 4), a result likely related to the larger ligand concentration obtained with the incubation procedure. Interestingly we found that, although absorbance of Rfx in the LB film increases continuously up to saturation, fluorescence quenching is not linear for all Rfx concentrations studied, for example we observed decrease of fluorescence intensity of FKBP12 of $70 \%$ at $\operatorname{Rfx}=9 \times 10^{-7} \mathrm{M}$ but only a $20 \%$ 
fluorescence decrease for $R f x=9 \times 10^{-6} \mathrm{M}$. This result likely reflects the occurrence of $\mathrm{Rfx}$ molecular aggregates [12] that are unavailable for binding. Indeed, also previous studies from our group showed that Rifaximin aggregates in aqueous solution at a critical aggregation concentration (c.a.c.) $=7 \times 10^{-6} \mathrm{M}[10]$.

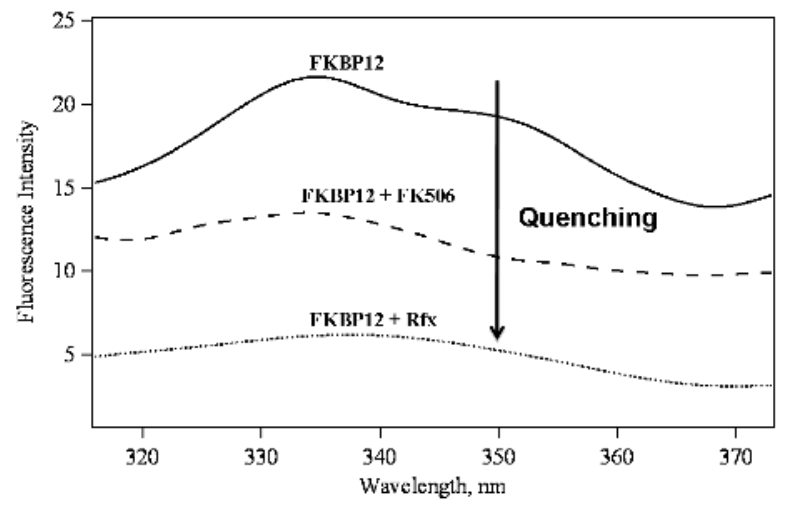

Fig. 4. Fluorescence emission spectra of FKBP12+Ligand free LB film (solid line), FKBP12+LB/FK506 (dashed line) obtained by co-spreading and FKBP12 LB/Rfx (dotted line) obtained by incubation with ligand. [FKBP12] $=1 \mu \mathrm{M}$.

Furthermore, fluorescence emission spectra of Rfx in LB film reported in figure 5 show the appearance of an emission band centred at $650 \mathrm{~nm}$ during and postincubation with FKBP12. Such band is absent for the $\mathrm{LB} / \mathrm{Rfx}$ system in buffer solution where a single band at $620 \mathrm{~nm}$ is present. The band splitting and the blue shift suggest a change in the molecular microenvironment of Rfx upon protein binding.

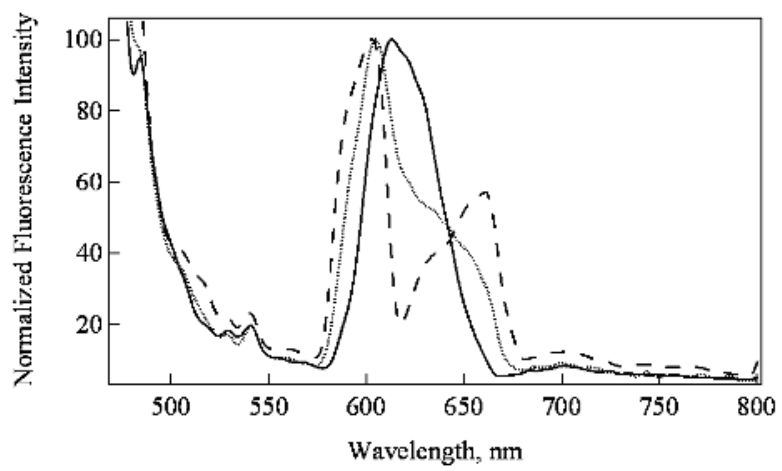

Fig. 5. Fluorescence emission spectra of Rfx in LB film in buffer solution (solid line), $\mathrm{Rfx}$ in LB film during incubation with FKBP12 (dashed line) and Rfx in LB film post-incubation with FKBP12 (dotted line). FKBP12 $=[1 \mu \mathrm{M}]$.

\section{Conclusions}

Solution quenching studies of the FKBP12-Rfx system evidenced how the commonly used Rfx antibiotic is very effective in binding the FKBP12 protein with a dissociation constant only two times larger than the $\mathrm{K}_{\mathrm{d}}$ found for Tacrolimus, the most widely used FKBP12 inhibitor. The body of the results also indicates that both Rfx and FK506 penetrate easily biomimetic systems such mono and bilayers of phospholipids although with different procedures. Incubation procedures yield good incorporation results for $\mathrm{Rfx}$ whereas co-spreading was demonstrated to produce LB structures incorporating FK506. Moreover, quenching studies proved that the binding to the FKBP12 protein was effective also when the ligands were immobilized in the LB films provided that the molecules were not aggregated. These results pave the way to important applications of these nanostructures, to name a few phospholipid formulation of liposomal systems for drug delivery as well as sensitive and specific nanosensors for FKBP12 for the early diagnosis of neurodegenerative amyloidosis. Studies of functionalized synthetic ligands [7] with surface-anchors and suitable spacers are currently in progress for the preparation of chemically modified surfaces [13] that can be directly coupled to sensing device for FKBP12.

\section{Acknowledgements}

M.R.M acknowledges MIUR (FIRB RBPR05JH2P Itananonet) for financial support.

\section{References}

1. W. Cao, M. Konsolaky, J. Biosci. 36 (3), pp 493-498 (2011).

2. M. Gerard, A. Deleersnijder, J. Demeulemeester, Z. Debyser, V. Baekelandt, Mol. Neurobiology, 44, 1327 (2011).

3. T. Al-Kayal, E. Russo, L. Pieri, G. Caminati, D. Berti, M. Bucciantini, M. Stefani, P. Baglioni, Soft Matter, 8, 9115-9126 (2012).

4. S. Morandi; M. Puggelli, G. Caminati, Colloids Surf. A Physicochem. Eng. Asp., 321,125-130 (2008).

5. M. Bizzarri, E. Tenori, M. R. Martina, S. Marsili, G. Caminati, S. Menichetti, P. Procacci, J. Phys. Chem. Lett. 2, 2834-2839 (2011).

6. C. H. Roehrig, C. Loch, J.-Y Siegal, M. Overhand, Chem. Med. Chem. 2, 1054-1070 (2007).

7. M.R Martina, E. Tenori, M. Bizzarri, S. Menichetti, G. Caminati, P. Procacci, J. Med. Chem. 56, 1041-1051 (2013).

8. C. Kozany, A. Maerz, C. Kress, F. Hausch, ChemBioChem, 10, 1402-1410 (2009).

9. O. Canadas, R. Guerriero, R. Garcia-Canero, G. Orellana, M. Menendez, C. Casals, Biochemistry, 43, 9926-9938 (2004).

10. S. Morandi, M. Puggelli, G. Caminati, Intern. J. Environ. Anal. Chem. 85, 665-673 (2005).

11. K.Roberts, Langmuir-Blodgett films, Plenum Press, NY 1990

12. S. Martini, A. Magnani, P. Corti, G. Corbini, R. Lampariello, M. P. Picchi, M. Ricci, C. Bonechi. Spectrosc. Lett., 35, 581 (2002).

13. F. Gambinossi, L. Lorenzelli, P. Baglioni, G. Caminati, Colloids Surf. A Physicochem. Eng.Asp., 321, 87-93 (2008) 